\title{
DETERMINAÇÃO DA COMPOSIÇÃO MINERAL DE DIFERENTES FORMULAÇÕES DE MULTIMISTURA
}

\author{
Vanessa Elias VIZEU Márcia Barreto S. FEIJÓ’, \\ Reinaldo Calixto de CAMPOS
}

\begin{abstract}
RESUMO
A multimistura como um produto, vem sendo largamente difundida no Brasil, sendo sistematizada, primeiramente pela Dra . Clara Brandão e implementada pela Pastoral da Criança da CNBB, associada as suas ações na área de atenção básica à saúde. Sua formulação varia de acordo com a disponibilidade local dos ingredientes, mas basicamente é composta de alimentos não convencionais na dieta habitual, entre eles os farelos, pó de casca de ovo, folhas verde-escuras e sementes. É um produto considerado como complemento nutricional, visando suprir deficiências nutricionais, principalmente em crianças desnutridas. No entanto, a biodisponibilidade de seus nutrientes e presença de fatores antinutricionais têm gerado polêmica e oferecido restrições ao seu emprego como tal. O presente estudo teve como objetivo avaliar a composição mineral (Ca, $\mathrm{Cu}, \mathrm{Fe}, \mathrm{K}, \mathrm{Mg}, \mathrm{Mn}, \mathrm{P}, \mathrm{Zn})$ de diferentes formulações de multimistura, visando conhecer o seu perfil como complemento nutricional de minerais, para crianças menores de cinco anos de idade. Diante dos resultados obtidos, observou-se que o teor de cinzas (resíduo mineral fixo) em $80 \%$ das amostras analisadas estavam abaixo do valor minimo preconizado no Regulamento Técnico para Fixação de Identidade e Qualidade de Mistura à Base de Farelos de Cereais e que a dose recomendada de multimistura, para essa faixa etária, não atinge o valor percentual da IDR que a qualificaria como complemento nutricional para esses minerais.
\end{abstract}

Palavras-chave: multimistura; alimentação alternativa; minerais; complemento nutricional.

\section{SUMMARY}

MEASUREMENT OF THE MINERAL COMPOSITION OF VARIOUS "MULTIMISTURA" FORMULATIONS. The "multimistura" is a broad distributed product in Brazil, first formulated by Dr. Clara Brandão and introduced by the CNBB (National Conference of Brazil Bishops), Children Pastoral as part of its activities in the health basic attention area. Its formula varies according to local ingredient availability but basically is compounded by non-conventional habitual diet kinds of food, among them are bran, egg shelf powder, dark green leaves and nuts and seeds. It is considered as a nutritional complement used to supply nutritional deficiencies mostly in underweight children. However, a controversy was created due to the real bioavailability of its nutrients and the presence in it of non-nutritional factors. These factors have restricted its use in this way. This research had as an objective measure the mineral composition ( $\mathrm{Ca}, \mathrm{Cu}, \mathrm{Fe}, \mathrm{K}, \mathrm{Mg}, \mathrm{Mn}, \mathrm{P}, \mathrm{Zn})$ of various "multimistura" formulations, by the espectrophotometry of atomic absorption, in a way to know its profile as a mineral nutritional complement for children up to five years old. By the results obtained it was seen that, in $80 \%$ of the samples, the ash content (fixed mineral rest) was below the minimum value expected in its Technical Rules for Identity Fixation and Quality of Mixtures Based in Cereal Bran. (RDC n. 53, 2000) where, the recommend dose for the "multimistura" in this age range does not signoficantly reache the DRI (Daily Recommended Ingestion). By this way, the formulations studied cannot be used as a nutritional complement for these minerals.

Keywords: multimistura; alternative food; minerals; nutritional complement.

\section{1 - INTRODUÇÃO}

Segundo dados da Pesquisa Nacional em Demografia e Saúde (PNDS) de 1996, 5,7\% das crianças brasileiras menores de cinco anos de idade são desnutridas. A desnutrição energético-protéica constitui uma das principais carências nutricionais no Brasil, com elevada prevalência na população de baixa renda. Os indivíduos mais vulneráveis por suas exigências nutricionais são as crianças, cujo desenvolvimento físico e mental está condicionado ao estado de nutrição dos primeiros anos de vida [21]. A desnutrição é a principal responsável pela alta taxa de mortalidade de crianças menores de cin-

Recebido para publicação em 05/05/2003. Aceito para publicação em 13/01/2005(001127).

Programa de Graduação em Nutrição, Universidade do Rio de Janeiro (UNIRIO). E-mail:vanessa.vizeu@superig.com.br

Departamento de Tecnologia de Alimentos, Universidade do Rio de Janeiro (UNIRIO).

Departamento de Química, Pontificia Universidade Católica do Rio de Janeiro (PUC-Rio).

A quem a correspondência deve ser enviada. co anos no Brasil e os índices de mortalidade infantil, apesar de virem reduzindo nas últimas décadas, ainda são altos.

Em 1976, os Drs. Clara e Rubens Brandão iniciaram, em Santarém, Pará, um programa para enfrentar a desnutrição, procurando na produção local "alimentos alternativos" que tivessem alto valor nutritivo, embora não fossem tradicionalmente consumidos pela população. Desta maneira, começaram a experimentar o uso de farelos e folhas verde escuras e, pouco a pouco, foram incorporando outros ingredientes como sementes e pó da casca de ovo. Após testarem a "alimentação alternativa", como ficou conhecido o programa, nas creches que atendiam, observaram uma redução drástica de diarréia entre as crianças, concluindo ser a multimistura crucial para os resultados de recuperação de desnutridos $[7,8,15]$. Em 1984, o Fundo das Nações Unidas para a Infância (UNICEF) designou um consultor para avaliar o programa, tendo sido seu relatório favorável quanto ao valor nutricional do mesmo. Em função destes fatos, a Pastoral da Criança, da CNBB, passou a difundir o uso da multimistura, associado as suas ações na área de atenção bási- 
ca à saúde, ganhando a divulgação dessa prática dimensões nacionais. Em 1989, com a transferência dos Drs. Clara e Rubens Brandão para o Ministério da Saúde, o programa de alimentação alternativa obteve ainda maior visibilidade.

Em 1992, o Instituto Nacional de Alimentação e Nutrição (INAN) reconheceu o potencial de utilização de algumas alternativas alimentares na recuperação e manutenção do estado nutricional de crianças e gestantes. Entretanto, ressaltou que os trabalhos existentes ainda não eram suficientes para afirmar com segurança o impacto produzido, o que indicava a necessidade de uma avaliação mais criteriosa. Assim sendo, foi criado, em 1994, um grupo de trabalho coordenado pelo presidente do INAN, também integrado pela Dra. Clara Brandão. O grupo tinha como objetivo elaborar um relatório que representasse um consenso de profissionais da área sobre o tema alimentação alternativa. A primeira questão a ser tratada seria a inclusão da alimentação alternativa nos programas do Ministério da Saúde [27, 29].

Em julho de 2000, foi criado o Regulamento Técnico para Fixação de Identidade e Qualidade a que deve obedecer a Mistura à Base de Farelo de Cereais. Nele, a multimistura fica definida como o produto obtido através da secagem, torragem, moagem e mistura de ingredientes de origem vegetal, sendo obrigatória a presença de farelos torrados em quantidade mínima de $70 \%$ (g/100g) e pó de folhas verde-escuras, podendo ser adicionados leite em pó e outros ingredientes [13].

Devido à grande polêmica gerada sobre a alimentação alternativa, vários autores dedicaram-se ao estudo do valor nutricional da multimistura. ASSIS et al. [3], por exemplo, avaliaram o impacto da complementação da dieta de crianças de 1 a 7 anos com farelo de trigo, um dos componentes principais da multimistura. Concluíram, após seis meses de estudo, que esta complementação não mostrava impacto significativo sobre o estado nutricional das crianças, segundo os indicadores antropométricos altura/idade, peso/idade e peso/altura. Já PRADO et al. [24] avaliaram o efeito da complementação da dieta com o farelo de trigo, na dose de $10 \mathrm{~g} / \mathrm{dia}$, na recuperação da anemia ferropriva em crianças de 1 a 6 anos de idade. Após seis meses de estudo, concluíram, através de avaliação bioquímica, que tal complementação não promoveu a recuperação da anemia nutricional nestas crianças. Semelhantes observações foram relatadas por LIMA et al. [19] que avaliaram, durante dois meses, o impacto da complementação da dieta com uma multimistura sobre o estado nutricional de crianças em fase préescolar de duas creches. Observaram que não houve alteração do estado nutricional das crianças em relação à antropometria e dosagens bioquímicas, concluindo que os resultados obtidos não mostraram alteração significativa com o uso da multimistura. RIBEIRO et al. [26] determinaram o teor de tiocianato urinário em crianças de 0 a 4 anos que utilizavam a multimistura como complemento nutricional. Tal determinação é útil no controle da exposição ao cianeto, presente na folha de mandioca e tó- xico aos seres humanos. O grupo que utilizava a multimistura apresentou niveis de excreção de tiocianato cerca de duas vezes maior que o grupo controle, revelando maior exposição ao cianeto. Estudos realizados com animais de experimentação $[6,8,16,17,18,30]$ não lograram valor nutricional da multimistura ou de seus componentes principais.

Quanto aos ingredientes utilizados, ALMEIDA et al. [1], avaliando a qualidade microbiológica e microscópica de farelo de trigo utilizado na composição de multimisturas, constataram a presença, acima do padrão estabelecido, de bolores, leveduras, Bacillus cereus e bactérias mesófilas, em cerca de 48, 40, 13 e 40\% do total das amostras analisadas, respectivamente. Tais resultados foram indicadores, portanto, de qualidade higiênica insatisfatória. Já na avaliação do uso do farelo de arroz, alguns lotes mostraram a presença de aflatoxina, um composto carcinogênico altamente resistente à destruição pelo calor de tostagem. Além disso, elevadas concentrações de ácido fitico, um fator antinutricional responsável pela diminuição da biodisponibilidade de minerais, foram encontradas. Os valores alcançaram teores de 5 a $6 \%$, um dos mais altos indices já referidos na literatura para alimentos [5].

$\mathrm{O}$ avanço do conhecimento sobre determinantes do crescimento e ganho de peso em crianças tem destacado o papel dos micronutrientes [3]. Sabe-se que os minerais desempenham diversas funções no organismo, atuando entre outros como cofatores enzimáticos, sendo requeridos em quantidades que dependem da fase de crescimento, das condições fisiológicas e do estado nutricional e de saúde do indivíduo [22]. Considerando as controvérsias ainda existentes quanto ao uso da multimistura como complemento nutricional, o presente estudo teve como objetivo determinar os teores de cálcio, cobre, ferro, potássio, magnésio, manganês, fósforo e zinco, de diferentes formulações de multimistura, a fim de se conhecer o perfil desses alimentos como complemento nutricional desses minerais para crianças menores de cinco anos.

\section{2 - MATERIAL E MÉTODOS}

Foram coletadas amostras representativas de multimistura utilizadas em cinco diferentes localidades dos municípios de Duque de Caxias e Petrópolis, no Rio de Janeiro.

\section{1 - Formulações das multimisturas}

Como a formulação da multimistura varia de acordo com a disponibilidade local dos ingredientes, na Tabela 1 está disposta a formulação de cada multimistura analisada.

\section{2 - Preparo das multimisturas}

As multimisturas são preparadas de uma forma padrão, ou seja:

- o farelo, a farinha e o fubá são torrados, separadamen- 
TABELA 1 - Formulação das multimisturas

\begin{tabular}{lccccc}
\hline \multicolumn{1}{c}{ Componentes } & Localidade & Localidade & Localidade & Localidade & Localidade \\
& 1 & 2 & 3 & 4 & 5 \\
\hline Farelo de trigo & $84 \%$ & $40 \%$ & $42 \%$ & $24 \%$ & 4 copos \\
Farinha de trigo & - & - & - & $24 \%$ & - \\
Fubá de milho & - & $40 \%$ & $42 \%$ & $24 \%$ & 4 copos \\
Aveia em flocos & - & - & - & $5 \%$ & - \\
Folha de mandioca & $8 \%$ & $20 \%$ & $8 \% *$ & $5 \%$ & 3 copos** \\
Casca de ovo & $8 \%$ & - & - & $3 \%$ & - \\
Semente de abóbora & - & - & $8 \%$ & $5 \%$ & - \\
Semente de melancia & - & - & - & - & 1 copo \\
Semente de gergelim & - & - & - & - & 5 colheres \\
Leite em pó integral & - & - & - & $10 \%$ & - \\
\hline
\end{tabular}

* Nessa localidade, a multimistura é feita com $8 \%$ de folhas (mandioca e couve)

** Nessa localidade, a multimistura é feita com 3 copos, de folhas (mandioca e batatadoce)

te, em panela de aluminio;

- as folhas são secas à sombra e depois liquidificadas;

- as sementes, quando presentes, são torradas no forno e depois liquidificadas;

- a casca de ovo, quando presente, é lavada, seca no forno e depois liquidificada;

- o leite em pó integral e a aveia em flocos, quando presentes, são adicionados no final da preparação.

\section{3 - Determinação da composição mineral}

Todas as amostras de multimistura foram analisadas em triplicata. Para a obtenção das cinzas (resíduo mineral fixo), foi procedida a incineração das amostras em forno mufla, a $550^{\circ} \mathrm{C}$, segundo metodologia da AOAC [4]. A determinação do cálcio, cobre, ferro, potássio, magnésio, manganês, fósforo e zinco foi realizada por espectrofotometria de absorção atômica com chama, em um espectrofotômetro Varian Thectron AA5, com exceção do fósforo que foi determinado em espectrofotômetro UVVisivel Varian Modelo DMS-100. Antes da determinação, os materiais utilizados foram descontaminados em solução de ácido nítrico $10 \%$ por 24 horas. A validação do método foi realizada pela análise de materiais certificados de referência, havendo sempre boa concordância entre os valores encontrados e certificados.

\section{3 - RESULTADOS E DISCUSSÃO}

A Tabela 2 mostra, em relação à massa úmida, os teores médios de cinzas encontrados nas multimisturas estudadas.
Os resultados encontrados para o teor de cinzas variaram entre $2,70 \%$ a $6,48 \%$, estando 4 das 5 amostras analisadas abaixo do valor mínimo preconizado no Regulamento Técnico para Fixação de Identidade e Qualidade de Misturas à Base de Farelos de Cereais, que

TABELA 2 - Teores médios de cinzas (g/100g) das multimisturas $(n=3)$

\begin{tabular}{ccc}
\hline Localidade & \% de Cinzas & D.P.* \\
\hline 1 & 6,48 & 0,12 \\
2 & 3,61 & 0,05 \\
3 & 2,70 & 0,04 \\
4 & 2,88 & 0,11 \\
5 & 3,36 & 0,11 \\
\hline
\end{tabular}

D.P. - Desvio Padrão

é de 5,5\% [13].

De acordo com a Tabela 3, as multimisturas estudadas poderiam ser consideradas um alimento com alto teor mineral de cobre, ferro, potássio, magnésio, manganês, fósforo e zinco, visto que elas atingem mais de $30 \%$ da IDR por $100 \mathrm{~g}$ para esses minerais [10]. Entretanto, como a porção de multimistura recomendada para crianças menores de cinco anos é muito pequena (2 colheres de chá/dia), ela não atinge o mínimo necessário de $25 \%$ da IDR para os minerais estudados, para ser considerada um complemento nutricional (Tabela 4), com exceção do manganês das localidades 1 e $2[9,11,12]$.

Além disso, o valor nutritivo de qualquer alimento não pode ser estabelecido unicamente com base na concentração de seus constituintes, uma vez que a sua qualidade nutricional também é determinada por uma série de fatores como equilíbrio entre seus constituintes, a forma quimica dos nutrientes, o tamanho das partículas, as interações entre diversos compostos da dieta, o estado fisiológico do indivíduo, as condições de processamento e de armazenagem e a ocorrência de fatores antinutricionais e inibidores [20].

No caso, o ácido fitico, encontrado nos farelos, prejudica a biodisponibilidade dos minerais. Sabe-se que um mol de ácido fitico pode quelar de 3 a 6 moles de cálcio, formando complexos insolúveis no $\mathrm{pH}$ intestinal. Esta mesma capacidade faz com que o ácido fitico forme também uma variedade de sais insolúveis com cátions di e trivalentes em $\mathrm{pH}$ neutro ( $\mathrm{Zn}, \mathrm{Cu}, \mathrm{Mn}, \mathrm{Fe}$ e $\mathrm{Mn})$, impedindo que estes minerais possam ser absorvidos no intestino $[23,28]$.

TABELA 3 - Composição mineral (mg/100g) das multimisturas estudadas

\begin{tabular}{|c|c|c|c|c|c|c|c|c|c|c|}
\hline \multirow[t]{2}{*}{ Minerais } & \multicolumn{2}{|c|}{ Localidade 1} & \multicolumn{2}{|c|}{ Localidade 2} & \multicolumn{2}{|c|}{ Localidade 3} & \multicolumn{2}{|c|}{ Localidade 4} & \multicolumn{2}{|c|}{ Localidade 5} \\
\hline & $(\mathrm{mg} / 100 \mathrm{~g})$ & D.P. & $(\mathrm{mg} / 100 \mathrm{~g})$ & D.P. & $(\mathrm{mg} / 100 \mathrm{~g})$ & D.P. & $(\mathrm{mg} / 100 \mathrm{~g})$ & D.P. & $(\mathrm{mg} / 100 \mathrm{~g})$ & D.P. \\
\hline $\mathrm{Ca}$ & 695,25 & 63,96 & 111,41 & 4,24 & 133,23 & 5,29 & 102,26 & 3,58 & 165,96 & 5,29 \\
\hline $\mathrm{Cu}$ & 1,38 & 0,21 & 0,79 & 0,02 & 0,71 & 0,06 & 0,45 & 0,05 & 0,41 & 0,02 \\
\hline $\mathrm{Fe}$ & 9,71 & 1,02 & 13,69 & 0,43 & 3,78 & 0,14 & 4,34 & 0,40 & 6,53 & 0,13 \\
\hline $\mathrm{K}$ & 1404,08 & 105,01 & 751,49 & 20,56 & 547,44 & 39,5 & 622,56 & 42,94 & 724,60 & 61,28 \\
\hline $\mathrm{Mg}$ & 449,97 & 23,99 & 269,25 & 6,33 & 168,28 & 4,43 & 153,05 & 12,46 & 269,04 & 11,56 \\
\hline $\mathrm{Mn}$ & 12,19 & 0,33 & 11,01 & 0,30 & 4,00 & 0,09 & 4,97 & 0,44 & 7,01 & 0,40 \\
\hline $\mathrm{P}$ & 1203,49 & 41,32 & 1307,80 & 30,76 & 412,36 & 9,10 & 501,51 & 24,76 & 596,85 & 8,87 \\
\hline $\mathrm{Zn}$ & 7,70 & 0,38 & 5,83 & 0,17 & 4,11 & 0,09 & 3,62 & 0,21 & 6,12 & 0,14 \\
\hline
\end{tabular}


TABELA 4 - Informação nutricional das multimisturas

Informação Nutricional

*Porção $=5 \mathrm{~g}(2$ colheres (chá) $/$ dia $)$

\begin{tabular}{|c|c|c|c|c|c|c|c|c|c|c|}
\hline & \multicolumn{2}{|c|}{ Localidade 1} & \multicolumn{2}{|c|}{ Localidade 2} & \multicolumn{2}{|c|}{ Localidade 3} & \multicolumn{2}{|c|}{ Localidade 4} & \multicolumn{2}{|c|}{ Localidade 5} \\
\hline & $\begin{array}{l}\text { Quant./ } \\
\text { porção }\end{array}$ & $\% \mathrm{IDR}^{* *}$ & $\begin{array}{l}\text { Quant./ } \\
\text { porção }\end{array}$ & $\%$ IDR & $\begin{array}{l}\text { Quant./ } \\
\text { porção }\end{array}$ & $\%$ IDR & $\begin{array}{l}\text { Quant./ } \\
\text { porção }\end{array}$ & $\%$ IDR & $\begin{array}{l}\text { Quant./ } \\
\text { porção }\end{array}$ & $\%$ IDR \\
\hline $\mathrm{Ca}(\mathrm{mg})$ & 34,8 & 4,3 & 5,6 & 0,7 & 6,7 & 0,8 & 5,1 & 0,6 & 8,3 & $\overline{1,0}$ \\
\hline $\mathrm{Cu}$ (mg) & 0,1 & 4,6 & 0,04 & 2,6 & 0,04 & 2,4 & 0,02 & 1,5 & 0,02 & 1,4 \\
\hline $\mathrm{K}(\mathrm{mg})$ & 70,2 & 5,0 & 37,6 & 2,7 & 27,4 & 2,0 & 31,1 & 2,2 & 36,2 & 2,6 \\
\hline $\mathrm{Mg}(\mathrm{mg})$ & 22,5 & 18,7 & 13,5 & 11,2 & 8,4 & 7,0 & 7,7 & 6,4 & 13,5 & 11,2 \\
\hline $\mathrm{Mn}(\mathrm{mg})$ & 0,6 & 30,5 & 0,6 & 27,5 & 0,2 & 10,0 & 0,2 & 12,4 & 0,4 & 17,5 \\
\hline $\mathrm{P}(\mathrm{mg})$ & 60,2 & 7,5 & 65,4 & 8,2 & 20,6 & 2,6 & 25,1 & 3,1 & 29,8 & 3,7 \\
\hline
\end{tabular}

* Porção recomendada por BRANDÃO \& BRANDÃO [9] para crianças até 5 anos de idade.

** IDR = Ingestão Diária Recomendada. Considerou-se a IDR para crianças até 5 anos de idade

Mais ainda, interações intraluminais por combinação química entre componentes da fibra da dieta, presentes na multimistura, e minerais como o ferro, cálcio, zinco, cobre ou magnésio são freqüentemente relatadas. Está propriedade, denominada troca catiônica, é responsável pelo aumento da excreção fecal de minerais e têm efeitos adversos sobre a utilização destes nutrientes. As conseqüências negativas sobre o estado nutricional dos indivíduos poderão ser significativas, particularmente para aqueles individuos que apresentam deficiência marginal de minerais [14, 25]

Com base nos conhecimentos atuais acerca da interação cálcio-ferro, um aumento na ingestão de cálcio pode resultar na instalação de um quadro de anemia em indivíduos que tenham uma ingestão marginal de ferro. Dentre os sais que interferem na biodisponibilidade de ferro sabe-se que o carbonato de cálcio, presente em grande quantidade na casca do ovo, é o responsável por maior redução da biodisponibilidade do ferro [14]. A casca do ovo, portanto, não deveria ser utilizada na formulação da multimistura, visto que um dos objetivos desta última é o de prevenir e curar a anemia nutricional. Para adequada complementação de cálcio na dieta, este deverá ser ingerido de 2 a 4 horas antes das refeições principais segundo ARAÚJO \& ARAÚJO [2], não interferindo, deste modo, na biodisponibilidade do ferro presente em maior quantidade nestas refeições.

\section{4 - CONCLUSÕES}

Apesar das multimisturas estudadas possuírem alto teor mineral por $100 \mathrm{~g}$, elas não atingem a IDR de referência para crianças menores de cinco anos, na dose recomendada, com exceção para o manganês das multimisturas das localidades $1 \mathrm{e} 2$. As multimisturas estudadas, portanto, se mostraram incapazes de servirem como complemento nutricional para os minerais quantificados nesse estudo. A pesquisa científica tem contribuído significativamente para o fortalecimento da segurança alimentar, entretanto, no tocante à alimentação alternativa, os estudos realizados com relação à utilização da multimistura nos últimos anos ainda têm sido poucos para garantir um nível de seguridade nutricional e toxicológica da mesma.

\section{5 - REFERÊNCIAS BIBLIOGRÁFICAS}

[1] ALMEIDA, N.R.; OLIVEIRA, I.C.; TAVARES, M.F.A.; SANTOS, G.M.S.; MONTAL, M.C.C.; OLIVEIRA, L.M.F. Avaliação da qualidade microbiológica e microscópica do farelo de trigo destinado à alimentação. Revista Baiana de Saúde Pública, Salvador, v. 19, n.1/4, p.9-18, 1992.

[2] ARAÚJO, A.C.M.F.; ARAÚJO, W.M.C. Cálcio e ferro: aspectos nutricionais. Higiene Alimentar, São Paulo, v.16, n.98, p.18-28, 2002.

[3] ASSIS, A.M.O.; PRADO, M.S.; FRANCO, V.B.; CONCEIÇÃO, L.H.; MARTINEZ, L.M.; OLIVEIRA, A.G. Suplementação da dieta com farelo de trigo e o estado nutricional de crianças de 1 a 7 anos de idade. Revista de Nutrição, Campinas, v.9, n.1, p.92-107, 1996.

[4] ASSOCIATION OF OFFICIAL ANALYTICAL CHEMISTS (AOAC). Official Methods of Analysis. 14. ed. Virginia, 1984. 1141p.

[5] BENEVIDES, C.M.J.; FURTUNATO, D.M.N.; OLIVEIRA, N. Alimentação alternativa: aspectos sócio-econômiconutricicionais. Higiene Alimentar, São Paulo, v.14, n.72, p. 13-16, 2000.

[6] BION, F.M.; PESSOA, D.C.N.P.; LAPA, M.A.G.; CAMPOS, F.A.C.S.; ANTUNES, N.L.M.; LÓPEZ, S.M.L. Uso de uma multimistura como suplementação alimentar: estudo em ratos. Archivos Latinoamericanos de Nutrición, Guatemala, v.47, n.3, p.242-247, 1997.

[7] BITTENCOURT, S.A. Uma alternativa para a política nutricional brasileira? Caderno de Saúde Pública, Rio de Janeiro, v. 14, n.3, p.629-636, 1998.

[8] BOAVENTURA, G.T.; CHIAPPINI, C.C.J.; FERNANDES, N.R.A.; OLIVEIRA, E.M. Avaliação da qualidade protéica de uma dieta estabelecida em Quissamã, Rio de Janeiro, adicionada ou não de multimistura e de pó de folha de mandioca. Revista de Nutrição, Campinas, v.13, n.3, p.201-209, 2000.

[9] BRANDÃO, C.T.; BRANDÃO, R.F. Alimentação Alternativa. Brasília: Centro de Pastoral Popular, 1996. 68p.

[10] BRASIL, Ministério da Saúde. Portaria no 27/98, Regulamento Técnico Referente à Informação Nutricional Complementar. Secretaria de Vigilância Sanitária. D.O.U., 13 de janeiro de 1998.

[11] BRASIL, Ministério da Saúde. Portaria no 32/98, 
Regulamento Técnico para Fixação de Identidade e Qualidade de Suplementos Vitamínicos e ou de Minerais. Secretaria de Vigilância Sanitária. D.O.U., 13 de janeiro de 1998.

[12] BRASIL, Ministério da Saúde. Portaria no 33/98. Ingestão Diária Recomendada (IDR) para Proteinas, Vitaminas e Minerais. Secretaria de Vigilância Sanitária. D.O.U., 30 de março de 1998.

[13] BRASIL, Ministério da Saúde. Resolução no 53/00. Regulamento Técnico para Fixação de Identidade e Qualidade de Misturas à Base de Farelo de Cereais. Secretaria de Vigilância Sanitária. D.O.U., 19 de junho de 2000.

[14] COELHO, R.G. Interações nutricionais / parte 1: interações ao nivel do trato gastrointestinal. Revista de Metabolismo e Nutrição, Porto Alegre, v.2, n.3, p.106$117,1995$.

[15] FARFAN, J.A. Alimentação alternativa: análise crítica de uma proposta de intervenção nutricional. Cadernos de Saúde Pública, Rio de Janeiro, v.14, n.1, p.205-212, 1998.

[16] FERREIRA, H.S.; SEARA, L.T; SILVA, A.M.D; PAES, C.A.M.C. Efetividade de uma multimistura como suplemento da dieta básica regional. Anais da Sociedade Brasileira de Ciências e Tecnologia de Alimentos, Campinas, v. 1, 1998.

[17] HEINEMANN, R.B.; COSTA, N.M.B.; CRUZ, R.; PIROZI, M.R. Valor nutricional da farinha de trigo combinada com concentrado protéico de folha de mandioca. Revista de Nutrição, Campinas, v.11, n.1, p.51-57, 1998.

[18] LEITE, M.S.; AZEREDO, V.B.; CARMO, M.G.T.; BOAVENTURA, G.T. Utilização da multimistura durante a lactação e seus efeitos na produção e composição do leite materno de ratas. Revista de Nutrição, Campinas, v. 15, n.2, p.211-221, 2002.

[19] LIMA, A.F.; COSTA, M.J.C.; OLIVEIRA, M.S.; CRUZ, A.C.S.; COSTA, A.L.P.; AIRES, J.S.; OLIVEIRA, K.V.S.; FREITAS, M.T.V. Estudo do impacto da dieta suplementada com multimistura sobre o estado nutricional de crianças em fase pré-escolar. Anais do VIII ENIC, João Pessoa, v.9, 1999.

[20] MILES, R.D.; HENRY, P.R. Relative trace mineral bioavailability. Ciência Animal Brasileira, Goiânia, v.1, n.2, p.73-93, 2000.
[21] MINISTÉRIO DA SAÚDE. Situação de saúde da criança brasileira. Disponivel em:

<http://www.saúde.gov.br/sps/areastecnicas/scrianca /crianca/situacao.htm>. Acesso em: 24/01/2003.

[22] ORGANIZAÇÃO MUNDIAL DE SAÚdE (OMS). Elementos Traço na Nutrição e Saúde Humanas. São Paulo: Livraria Roca, 2002. 316p.

[23] PAYÁ, J.B.; MONTORO, A.V.; PAYÁ A.B. Los enzimas en nutrición porcina (II). Disponivel em:

<http://www.revista-anaporc.com/enzimar2.htm>. Acesso em: 05/12/2002.

[24] PRADO, M.S.; ASSIS, A.M.O.; FRANCO, V.B.; ARAÚJO, M.P.N.; SILVA, A.; FARIA, J.A.; MARTINS, M.C. Suplementação da dieta com farelo de trigo e recuperação da anemia em crianças de 1 a 6 anos de idade. Revista de Nutrição, Campinas, v. 8, n.1, p.145-163, 1995.

[25] RAUPP, D.S.; MARQUES, S.H.P.; ROSA, D.A.; CALDI, C.M.; CREMASCO, A.C.V.; BANZATTO, D.A. Arraste via fecal de nutrientes da ingestão produzido por bagaço de mandioca hidrolisado. Scientia Agricola, São Paulo, v.59, n.2, p.235-242, 2002.

[26] RIBEIRO, M.R.; LUCAS, A.C.S.; SANTOS, P.D.; GALVÃO, J.F. Teor de tiocianato urinário em crianças de 0 a 4 anos que utilizam alimentação alternativa (multimistura). Revista da Universidade do Amazonas Série Ciências da Saúde, v.4/5, n.1/2, p.95-110, 1996.

[27] SANTOS, L.A.S; LIMA, A.M.P.; PASSOS, L.M.P.; SOARES, M.D.; SANTOS, S.M.C. Uso e percepções da alimentação alternativa no estado da Bahia: um estudo preliminar. Revista de Nutrição, Campinas, v.14, suplemento, p.35-40, 2001

[28] SILVA, M.R.; SILVA, M.A.A.P. Aspectos nutricionais de fitatos e taninos. Revista de Nutrição, Campinas, v.12, n.1, p.21-32, 1999.

[29] VELHO, L; VELHO, P. A controvérsia sobre o uso de alimentação alternativa no combate à subnutrição no Brasil. História Ciências, Saúde Manguinhos, Rio de Janeiro, v.9, n.1. p.125-57, 2002.

[30] VIANA, A.J.B. Aspectos histológicos de células do cólon de ratos wistar suplementados com multimistura. João Pessoa, 2001. 87p. Dissertação (Mestrado em Ciências da Nutrição) Faculdade de Ciências da Nutrição, Universidade Federal da Paraíba. 